\title{
Pump Down the Prep to Pump Up the Uptake: Dream or Nightmare?
}

\author{
Raf Bisschops ${ }^{1}$, Cesare Hassan ${ }^{2}$
}

1 Department of Gastroenterology and Hepatology, University Hospitals Leuven, Leuven, Belgium

2 Endoscopy Unit, Nuovo Regina Margherita Hospital, Rome, Italy 
Colorectal Cancer (CRC) screening of average-risk subjects represents one of the most universally strong recommendation adopted in any national or international guideline.[1] Unexpectedly, a substantial proportion of the target population does not participate in screening programs.[2] It could be argued that this is not specific for CRC screening and that it is related to personal disbelief in the risk of developing cancer and the efficacy of the recommended interventions or to attitudes as fatalism, denial of CRC risk and a low socio-economic status. Several interventions aiming to remove these barriers by engaging and motivating nonadherent subjects have been recommended with variable and generally disappointing results.[ 3]

For CRC screening however, there is an unique barrier that relates to the endoscopy community. Colonoscopy is still perceived as an invasive, painful screening procedure, generating irrational fears. Indeed, uptake is much higher for non-endoscopic (immunochemical faecal test) primary tests [4]. Implementation of sedation protocols to reduce pain can address this barrier, as illustrated by the high uptake of primary screening colonoscopy in the United States that was associated with a boost of anesthesiologist-assisted colonoscopies[5].

A second major barrier for uptake in a colonoscopy-based screening program is poor tolerability of bowel preparation. This is directly related to the large volume of an isotonic agent (Polyethylene Glycol (PEG)) to achieve an adequate cleansing of the colon without causing electrolyte and volume imbalances. This question has been studied in this issue of Endoscopy by a nationwide randomized trial in Poland, comparing the uptake to a primary screening colonoscopy when offering a high- versus a low-volume preparation regimen to average-risk eligible subjects that were previously not exposed to colonoscopy.[6] By analyzing 13,497 randomized subjects within the Polish screening program, the authors failed to show any increase in uptake when inviting the targeted population by offering either a highvolume $4 \mathrm{~L}$ PEG regimen or an ultra-low volume $(300 \mathrm{ml}$ sodium picosulfate/magnesium citrate) non-PEG solution, resulting in a $16.6 \%$ and $15.5 \%$ participation rate, respectively.[6]

This study clearly indicates that the volume of the bowel preparation in patients who never underwent a colonoscopy before is not the main determining factor to persuade patients to participate in a screening program. Thus, factors other than the volume of the cleansing regimens are responsible for the fact that roughly $85 \%$ of the eligible Polish population actually refused participation.[6] This does not necessarily preclude any effect between tolerability and 
screening uptake for surveillance endoscopy, but rather suggests the existence of a hierarchy between other technical and non-technical factors and acceptability. For instance, an unexpected $30 \%$ of colonoscopy-associated pain has been reported by the Polish subjects undergoing screening colonoscopy.[7] This data is much more likely to affect the acceptability of colonoscopy rather than the volume of laxative. Secondly, the mail-based invitation system of the Polish program may be insufficient to address psychological or organizational barriers that prevent most of the people to accept the recommended intervention. For instance, older age, male sex and travel distance from the screening centres appeared to predict participation, irrespectively of the chosen regimen.[6] It is also important to emphasize that the Polish data did not assess acceptability of the bowel preparation as such. It is conceivable that for the uptake for follow-up colonoscopy, this may play a crucial factor as indicated in clinical trials.[8] It would also be interesting to assess the patient's preference when oral instructions were to be given and the patient was offered to choose between bowel preparations.

The lack of increased uptake in the Polish trial does not mean that low-volume bowel preparations have not contributed to the evolution of colonoscopy. Meta-analysis of wellconducted randomized trials have shown improved tolerability for low-volume regimens with equal efficacy and safety.[9] Thus, if not as a facilitator for screening participation, volume reduction is still a technical improvement with a positive impact on patient-experience, one of the ESGE key performance measures for colonoscopy and endoscopy services.[10] The current study however underlines the difference between well controlled and monitored randomized trials, during which patients are well instructed on the use of a product, and a real life situation when asymptomatic individuals receive written instructions for their procedure. In view of the evidence of the trials, it emphasizes the importance to give clear instructions to motivate patients and also to select the correct patients for the correct bowel preparation. Indeed, chronic constipation is often an exclusion criterion for trials, but has a high prevalence in the general population.

With regard to quality of colonoscopy, the second main result of this Polish study is the difference in the quality of cleansing and adenoma detection rates between a high- and a lowvolume regimens when pragmatically implemented in a population-based screening program.[6] Unexpectedly, the present study showed the clear inferiority of the low-volume regimen in the level of adequate cleansing (79\% versus $86.6 \%$, respectively).[6] Bench-marked against an ESGE threshold of $90 \%$, the low-volume regimen is too far off target.[10] In addition, the level of optimal cleansing, as rated by the highest values of the BBPS, appeared 
to be better for the high-volume arm.[6] Even more worrisome, although less clear, is a higher detection rate, especially for proximal lesions, including advanced adenomas and serrated lesions in favor of the high volume bowel preparation.[6] This data overall indicates that before implementing a low-volume regimen in an organized program based on the results of highquality randomized trials, a pragmatic verification of its effectiveness by monitoring and auditing the main quality indicators is necessary in a real life setting where patients receive less rigid instructions and can be constipated.

In conclusion, the study by Pisera $\mathrm{M}$ et al. indicates that in colonoscopy naive patients who are only informed by written instructions, the volume of the bowel preparation is not the main determining factor to proceed with screening colonoscopy.[6] The low uptake of CRC screening in several European countries is unlikely to be reversed by a mere technical improvement of colonoscopy, but requires long-lasting complex and multidimensional interventions at the different levels of the national or regional health system.

1. von Karsa L, Patnick J, Segnan $\mathrm{N}$ et al. European guidelines for quality assurance in colorectal cancer screening and diagnosis: overview and introduction to the full supplement publication. Endoscopy 2013; 45: 51-59

2. Senore C1 BP, Anttila A3, Ponti A1, Tomatis M1, Vale DB4, Ronco G1, Soerjomataram 15, Primic Zakelj M6, Riggi E1. Dillner J7, Elfstrom KM8, Lonnberg S9, Sankaranarayanan R10, Segnan N1. Performance of Colorectal cancer screening in the European Union Member States- Data from the second European screening report. DOI:

3. Hassan C, Kaminski MF, Repici A. How to Ensure Patient Adherence to Colorectal Cancer Screening and Surveillance in Your Practice. Gastroenterology 2018; 155: 252-257

4. Segnan N, Senore C, Andreoni B et al. Comparing attendance and detection rate of colonoscopy with sigmoidoscopy and FIT for colorectal cancer screening. Gastroenterology 2007; 132: 2304-2312

5. Adams MA, Prenovost KM, Dominitz JA et al. Predictors of Use of Monitored Anesthesia Care for Outpatient Gastrointestinal Endoscopy in a Capitated Payment System. Gastroenterology 2017; 153: 1496-1503 e1491

6. article FEirtte. DOI:

7. Bugajski $M$, Wieszczy $P$, Hoff $G$ et al. Modifiable factors associated with patient-reported pain during and after screening colonoscopy. Gut 2017, DOI: 10.1136/gutjnl-2017-313905:

8. Katz PO, Rex DK, Epstein $\mathrm{M}$ et al. A dual-action, low-volume bowel cleanser administered the day before colonoscopy: results from the SEE CLEAR II study. The American journal of gastroenterology 2013; 108: 401-409

9. Martel M, Barkun AN, Menard C et al. Split-Dose Preparations Are Superior to Day-Before Bowel Cleansing Regimens: A Meta-analysis. Gastroenterology 2015; 149: 79-88

10. Kaminski MF, Thomas-Gibson S, Bugajski $M$ et al. Performance measures for lower gastrointestinal endoscopy: a European Society of Gastrointestinal Endoscopy (ESGE) Quality Improvement Initiative. Endoscopy 2017; 49: 378-397 
